\title{
Acute aortic dissection developed after cardiopulmonary resuscitation: transesophageal echocardiographic observations and proposed mechanism of injury
}

\author{
Dong Keon Lee ${ }^{1}$, Kyung Sik Kang ${ }^{2}$, Yong Sung Cha ${ }^{2}$, Kyoung-Chul Cha ${ }^{2}$, Hyun Kim², Kang Hyun Lee ${ }^{2}$, \\ Sung Oh Hwang ${ }^{2}$ \\ 'Department of Emergency Medicine, Hallym University Sacred Heart Hospital, Hallym University College of Medicine, Anyang; ${ }^{2}$ Department of Emergency \\ Medicine, Yonsei University Wonju College of Medicine, Wonju, Korea
}

There has been no report about aortic dissection due to cardiopulmonary resuscitation (CPR). We present here a case of acute aortic dissection as a rare complication of CPR and propose the potential mechanism of injury on the basis of transesophageal echocardiographic observations. A 54-year-old man presented with cardiac arrest after choking and received 19 minutes of CPR in the emergency department. Transesophageal echocardiography (TEE) during CPR revealed a focal separation of the intimal layer at the descending thoracic aorta without evidence of aortic dissection. After restoration of spontaneous circulation, hemorrhagic cardiac tamponade developed. Follow-up TEE to investigate the cause of cardiac tamponade revealed aortic dissection of the descending thoracic aorta. Hemorrhagic cardiac tamponade was thought to be caused by myocardial hemorrhage from CPR.

Key Words: cardiopulmonary resuscitation; heart arrest; thoracic aortic aneurysm

\section{Case Report}

Received: November 20, 2015

Revised: May 17, 2016

Accepted: October 13, 2016

Corresponding author Sung Oh Hwang Department of Emergency Medicine, Yonsei University Wonju College of Medicine, 20 Ilsan-ro, Wonju 26426, Korea

Tel: +82-33-741-1611

Fax: +82-33-742-3030

E-mail: shwang@yonsei.ac.kr

External chest compression during cardiopulmonary resuscitation (CPR) may be traumatic. Complications from external chest compressions during CPR include bruising and abrasions of the skin, rib and sternum fractures, bone marrow embolism, cardiac injuries including myocardial hemorrhages and frothy heart blood, and injuries to abdominal organs such as the liver and spleen [1,2]. Injuries to the bony thorax are most the common complication [3]. The aorta is considered to be safe from traumatic injury because it is located deep in the chest and surrounded by bony structures. There have been few reports regarding aortic injury caused by CPR, possible because of the low incidence of injury to the aorta or the inability to detect this complication during CPR. Transesophageal echocardiography (TEE) provides an excellent window to the heart and the aorta and provides useful information about blood flow during CPR without interfering with resuscitation measures [4,5]. With TEE, aortic complications associated with CPR can be easily diagnosed.

Here we report a case of aortic dissection that developed after CPR, which was observed by TEE, and describe possible mechanisms of aortic injury on the basis of transesophageal echo-
Copyright $(\odot) 2019$ The Korean Society of Critical Care Medicine

This is an Open Access article distributed under the terms of Creative Attributions Non-Commercial License (http:// creativecommons.org/li-censes/by-nc/4.0/) which permits unrestricted noncommercial use, distribution, and reproduction in any medium, provided the original work is properly cited. 
cardiographic observation of the aorta during CPR in this case.

\section{CASE REPORT}

A 54-year-old man presented with cardiac arrest after choking on food at home. He had been suffering from dementia and right-sided weakness after a traumatic intracranial hemorrhage 7 years prior. He received immediate bystander CPR and was brought to our emergency department (ED). Asystole was documented at admission, and the resuscitation attempt was continued in the ED. After endotracheal intubation, a TEE transducer was inserted to find the cardiovascular cause of cardiac arrest. TEE revealed no cardiac contraction. No structural cardiac abnormality or pericardial effusion was noted. A focal separation of the intimal layer at the anterior wall of the descending thoracic aorta (DTA) was seen $10 \mathrm{~cm}$ from the distal portion of the aortic arch (Figure 1A). The DTA was compressed and deformed repetitively whenever the chest was compressed (Supplementary Video 1). After 19 minutes of resuscitation, the patient regained spontaneous
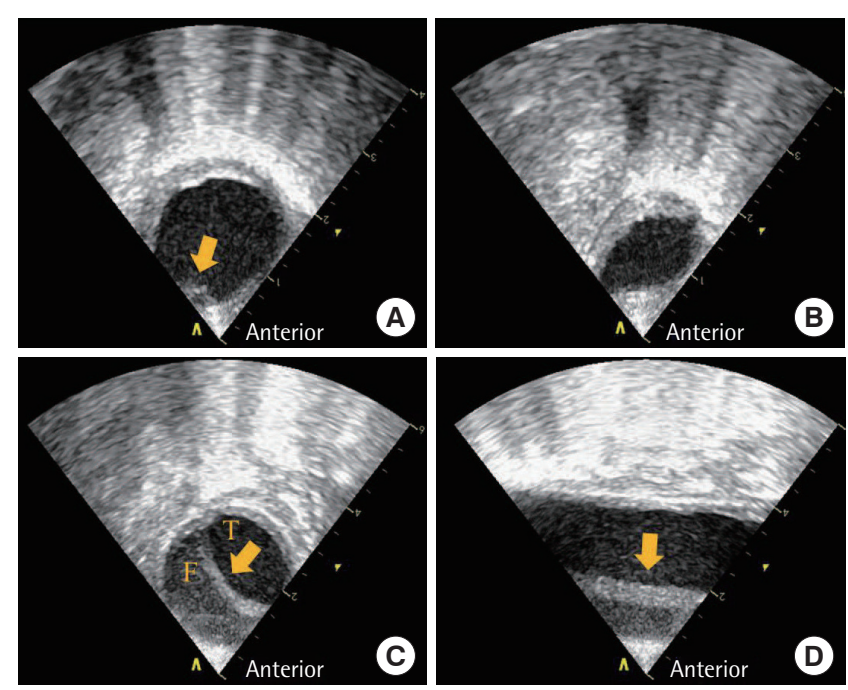

Figure 1. Transesophageal echocardiographic findings of the aorta. (A) Transesophageal echocardiography (TEE) findings in the aorta during cardiopulmonary resuscitation. A focal separation of the intimal layer at the anterior wall of descending thoracic aorta (DTA) with atherosclerotic changes (arrow) was seen on the short axis view of the aorta. (B) Compression of the DTA during compression systole. The DTA was compressed and deformed on the short axis view of the aorta when external chest compression was performed. (C) Short axis view of the aorta after restoration of spontaneous circulation (ROSC). Follow-up TEE revealed the intimal flap of the DTA (arrow) on the short axis view of the aorta. (D) Long axis view of the aorta after ROSC. The intimal flap (arrow) was observed between the distal portion of the aortic arch and 11 $\mathrm{cm}$ down from the aortic isthmus. T: true lumen; F: false lumen. circulation and the TEE transducer was removed. His systolic blood pressure rose to $130 \mathrm{~mm} \mathrm{Hg}$. Ten minutes after restoration of spontaneous circulation (ROSC), his systolic blood pressure dropped to $80 \mathrm{~mm} \mathrm{Hg}$. Transthoracic echocardiography showed a newly developed pericardial effusion with diastolic collapse of the right ventricle, suggesting cardiac tamponade. Bloody pericardial fluid was drained during emergency pericardiocentesis. Follow-up TEE was performed to investigate the origin of pericardial effusion. TEE revealed an intimal flap, not seen on the first TEE, in the DTA (Figure 1B). The intimal flap was observed between the distal portion of the aortic arch and $11 \mathrm{~cm}$ down from the aortic isthmus (Figure 1C and D, Supplementary Video 2). Thoraco-abdominal angiography with a multi-detector computed tomography (CT) scanner (Brilliance 64; Philips Medical Systems, Cleveland, OH, USA) revealed aortic dissection of the DTA with no involvement of the ascending and abdominal aorta (Figure 2). Bloody pericardial effusion was thought to be one of the complications from CPR because there was no evidence of dissection of the ascending thoracic aorta.

The patient was moved to the intensive care unit and received intravenous labetalol to control heart rate and blood pressure. Therapeutic hypothermia with intravascular cooling (Coolgard 3000 Intravascular Temperature Management system; Zoll Medical, Chelmsford, MA, USA) was performed. On the 4 th day of hospital admission, he remained comatose and
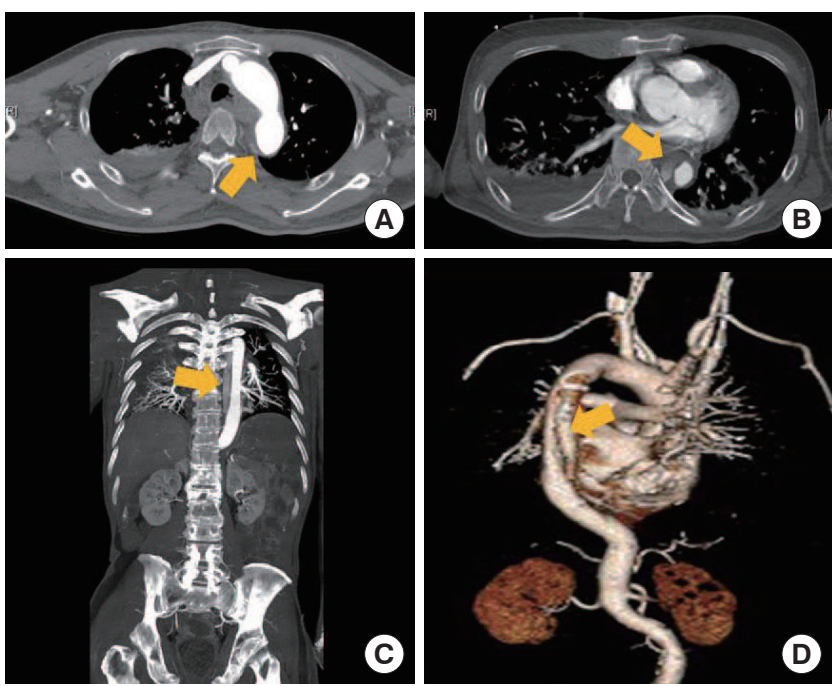

Figure 2. Thoraco-abdominal computed tomography (CT) angiographic findings. (A) Intimal tear in the proximal descending aorta (arrow). (B) Intimal flap in the dissected aorta (arrow). (C) Coronal view of chest CT angiography shows aortic dissection of the descending thoracic aorta (DTA) with no involvement of the ascending and abdominal aorta (arrow). (D) Three-dimensional CT angiography shows dissection of the DTA (arrow). 
his Pittsburgh Brainstem Score was 6. On the 5th day of hospital admission, an electroencephalogram showed diffuse slowing waves. On the 13th day of hospital admission, the patient died of severe hypoxic brain injury.

\section{DISCUSSION}

External chest compressions may produce blunt chest trauma due to the nature of forceful compression of the sternum. Aortic injury from blunt chest trauma is relatively uncommon, and is found in between $1.5 \%$ and $2 \%$ of patients who sustain blunt thoracic trauma [6-9]. There has been only one report regarding aortic injury due to CPR in the literature. Juan et al. [10] reported an intramural hematoma of the aorta found on a chest CT scan taken after CPR in a patient who received CPR and thrombolysis due to cardiac arrest from a pulmonary embolism. In their report, the direct relationship between resuscitation and aortic injury is unknown because no evaluation of the aorta could be made prior to or during CPR. We identified a direct relationship between resuscitation and aortic injury because aortic dissection, not seen on the first TEE, was observed on the second TEE after ROSC.

The thorax undergoes repetitive compression and relaxation during CPR. On the basis of our observation of the aorta during CPR, we propose that pinching and compression of the aorta was the possible mechanism of aortic injury developed from external chest compression.

Transesophageal echocardiographic observation during CPR shows that the heart is displaced toward the back and that the DTA at the maximal compression area is compressed and deformed during compression systole, as observed in this case [11]. This observation suggests that the descending aorta can be pinched between the displaced heart and the thoracic cage overlying the aorta. The exact mechanism of the aortic injury in this case remains unknown. However, the DTA might be injured from the pinch between the posterior thoracic wall and the heart during chest compression, deceleration by chest compression, or both. Repetitive compressions and/or decelerations of the aorta can cause aortic injury by exerting shearing force on the aortic wall, which results in separation of the intimal layer. In this case, aortic dissection began at the distal portion of the aortic arch and was observed over $11 \mathrm{~cm}$ down from the aortic isthmus. This finding supports the idea that repetitive pinching of the aorta and compression causing shearing force on a pre-existing tear may be involved in the mechanism of aortic dissection.

In conclusion, aortic dissection is an extremely rare com- plication of CPR. However, we should consider the possibility of aortic injury from CPR before assuming thoracic aortic dissection as one of the causes of cardiac arrest. TEE can be a useful means of early detection of aortic injury associated with CPR.

\section{CONFLICT OF INTEREST}

No potential conflict of interest relevant to this article was reported.

\section{ORCID}

$\begin{array}{ll}\text { Dong Keon Lee } & \text { https://orcid.org/0000-0003-0490-1837 } \\ \text { Kyung Sik Kang } & \text { https://orcid.org/0000-0002-1687-8593 } \\ \text { Yong Sung Cha } & \text { https://orcid.org/0000-0001-9897-4273 } \\ \text { Kyoung-Chul Cha } & \text { https://orcid.org/0000-0003-1818-2466 } \\ \text { Hyun Kim } & \text { https://orcid.org/0000-0002-1696-9401 } \\ \text { Kang Hyun Lee } & \text { https://orcid.org/0000-0001-9664-9186 } \\ \text { Sung Oh Hwang } & \text { https://orcid.org/0000-0003-4585-3181 }\end{array}$

\section{AUTHOR CONTRIBUTIONS}

Conceptualization: SOH. Data curation: DKL, KSK. Methodology: YSC, KCC. Project administration: SOH. Visualization: DKL, SOH. Writing - original draft: DKL. Writing - review \& editing: HK, KHL, SOH.

\section{SUPPLEMENTARY MATERIALS}

The online-only supplement data are available with this article online: https://doi.org/10.4266/acc.2015.00633.

\section{REFERENCES}

1. Hashimoto Y, Moriya F, Furumiya J. Forensic aspects of complications resulting from cardiopulmonary resuscitation. Leg Med (Tokyo) 2007;9:94-9.

2. Krischer JP, Fine EG, Davis JH, Nagel EL. Complications of cardiac resuscitation. Chest 1987;92:287-91.

3. Hoke RS, Chamberlain D. Skeletal chest injuries secondary to cardiopulmonary resuscitation. Resuscitation 2004;63:327-38.

4. Higano ST, Oh JK, Ewy GA, Seward JB. The mechanism of blood flow during closed chest cardiac massage in humans: transesophageal echocardiographic observations. Mayo Clin Proc 1990;65:1432-40.

5. Ma MH, Hwang JJ, Lai LP, Wang SM, Huang GT, Shyu KG, et al. Transesophageal echocardiographic assessment of mitral 
valve position and pulmonary venous flow during cardiopulmonary resuscitation in humans. Circulation 1995;92:854-61.

6. Dyer DS, Moore EE, Ilke DN, McIntyre RC, Bernstein SM, Durham JD, et al. Thoracic aortic injury: how predictive is mechanism and is chest computed tomography a reliable screening tool? A prospective study of 1,561 patients. J Trauma 2000;48: 673-82.

7. Mirvis SE, Shanmuganathan K, Buell J, Rodriguez A. Use of spiral computed tomography for the assessment of blunt trauma patients with potential aortic injury. J Trauma 1998;45: 922-30.

8. Steenburg SD, Ravenel JG. Acute traumatic thoracic aortic injuries: experience with 64-MDCT. AJR Am J Roentgenol 2008;
191:1564-9.

9. Ungar TC, Wolf SJ, Haukoos JS, Dyer DS, Moore EE. Derivation of a clinical decision rule to exclude thoracic aortic imaging in patients with blunt chest trauma after motor vehicle collisions. J Trauma 2006;61:1150-5.

10. Juan YH, Saboo SS, Desai NS, Khandelwal K, Khandelwal A. Aortic intramural hematoma and hepatic artery pseudoaneurysm: unusual complication following resuscitation. Am J Emerg Med 2014;32:107. e1-4.

11. Hwang SO, Lee KH, Cho JH, Yoon J, Choe KH. Changes of aortic dimensions as evidence of cardiac pump mechanism during cardiopulmonary resuscitation in humans. Resuscitation 2001;50:87-93. 